\title{
CLASHING CONSCIOUSNESS:
}

\section{A Cure for Modern Medicine's \\ Epistemic Privilege}

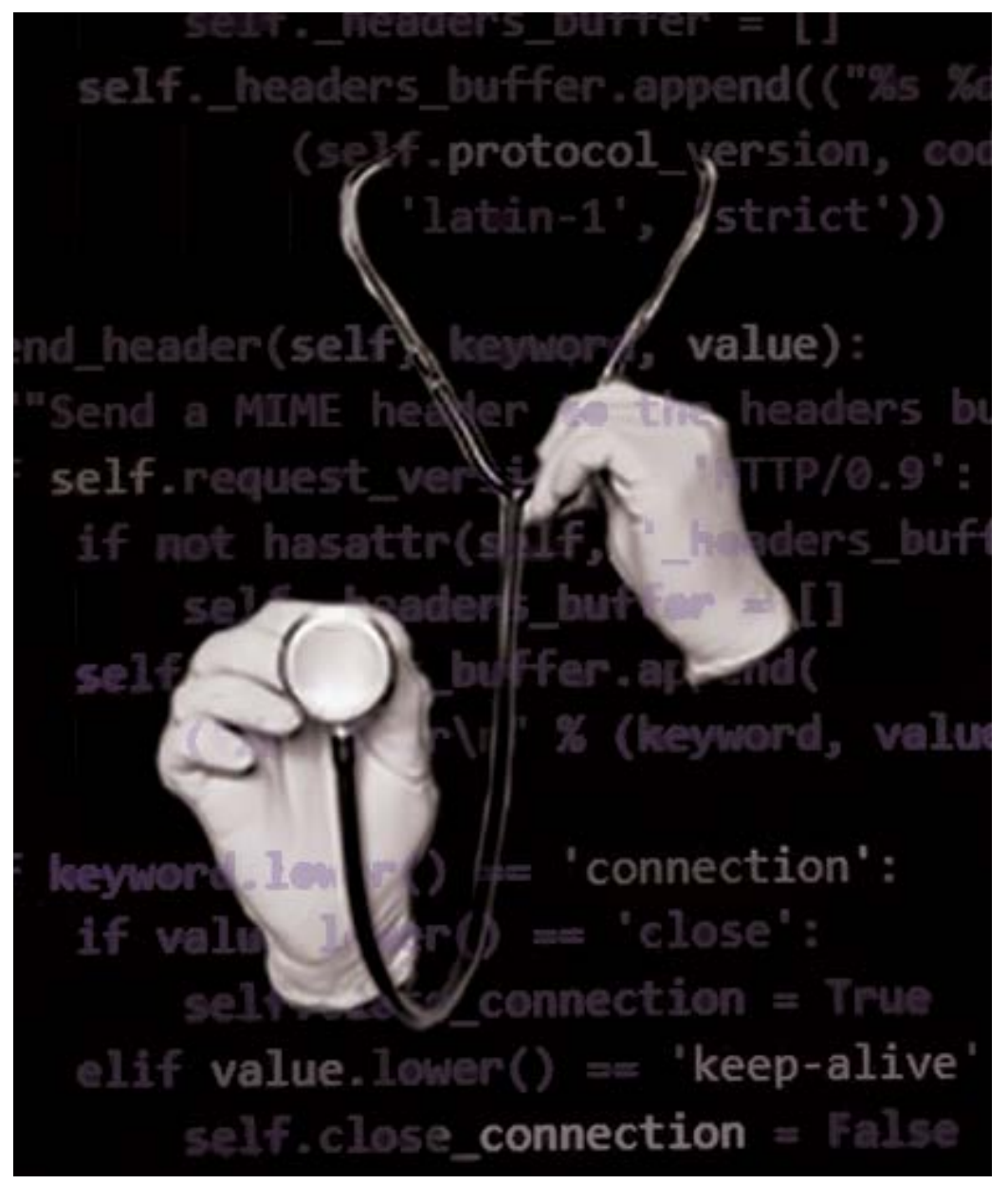

\section{ABSTRACT}

In this paper, I consider practical strategies for resolving the epistemic injustice that ill persons face when seeking medical treatment. My arguments will expand upon those initially made by Havi Carel and Ian James Kidd in "Epistemic Injustice in Healthcare: A Philosophical Analysis." My approach to this problem is twofold. First, I will demonstrate how the phenomenological toolkit, as it currently stands, emphasizes the patient's experience and leaves the doctor's experience unadjusted. After this, I will explain how the toolkit can be improved to include the doctor's perspective.

BRADLEY HOLDER 


\section{INTRODUCTION}

A hospital is, in many ways, an excellent microcosm of the society within which it exists. In the developed world, we see public interest colliding rather chaotically with private interest, we see highly trained and well-paid medical personnel scrambling to treat a perhaps unsustainable plethora of patients, and we see chronically ill persons, a special type of patient, whose schedule of visits to the doctor's office is not quite as terminal as the illness they probably possess, consistently overstepped and ignored. All this, and more, in the spirit of the scientific method. What is it exactly that causes and permits this overstepping, and what might we, as philosophers and medical professionals, do to fix it?

The issue stems primarily from the disproportionate values assigned to the knowledge of the doctor and the knowledge of the chronically ill patient. Because medicine is, in fact, an applied science, the level of importance granted to objectively acquired data, like lab results for example, always exceeds that of subjective testimony, such as the patient's feelings of pain or discomfort. This type of prejudice ensures that modern healthcare professionals (HCPs) regularly devalue the testimony of their patients, even when said testimony pertains to the patient's wellness plan, simply out of convention: "The patient can't possibly know more about their cancer than I do. They're just a patient. I'm the doctor." This sense of entitlement, acquired from, among other things, the social prestige of the position, is called epistemic privilege and is the source of the mistreatment of chronically ill persons. In the following, I will refer to this mistreatment as epistemic injustice.

The question remains, however: How can the study of philosophy rid the medical field of epistemic privilege and epistemic injustice? Something must be done beyond simply proving that patient testimony is useful. What needs to happen, then, is that doctors must be given the opportunity to properly see the patient not merely as an object but also as a subject. If the patient/doctor interaction can be altered to include a mandated and overseen shared experience, then compassion will prevail, and the significance of the patient's testimony will be reasserted.

In the following sections, I will provide a critical summary of Havi Carel and Ian James Kidd's essay, "Epistemic Injustice in Healthcare: A Philosophical Analysis," in addition to providing a counterargument to the authors' conclusion. I will demonstrate that while Kidd and Carel's "phenomenological toolkit," as it stands, may be useful against individual instances of epistemic injustice, unless it undergoes considerable revision, it will be unable to affect the much larger problem of epistemic privilege. ${ }^{1}$ The altered toolkit, after these revisions have been implemented, will include three steps: (1) bidirectional expression of intent, (2) formal mediation, and (3) posthumous review. I will explain the details as well as the significance of these steps at considerable length in the pages that follow.

\section{CRITICAL SUMMARY OF CAREL AND KIDD'S ARTICLE}

Havi Carel and Ian James Kidd, in "Epistemic Injustice in Healthcare: A Philosophical Analysis," explain the various types of epistemic injustice that occur within modern medical practice between doctors and patients. They attempt to determine the exact source of this epistemological imbalance-i.e., the medical practitioner's unquestioned authority over the patient - and ultimately provide a "phenomenological toolkit" to allow patients to express their concerns and beliefs regarding their respective illnesses. This expression of concern is supposed to enable the patient to experience a type of catharsis; furthermore, the doctor, by virtue of having witnessed this expression, can better sympathize with the patient's illness.

The authors divide epistemic injustice into two broad categoriestestimonial injustice and hermeneutical injustice - the former referring to instances where a patient's testimony is devalued (i.e., not believed) and the latter referring to instances where a patient is not given the opportunity or ability to communicate properly with their doctor.

Epistemic privilege is the mechanism by which ill persons experience epistemic injustice. The healthcare industry, as an archetype of society, encourages a delimiting hierarchy of values, including, but not limited to, certain modes of communication. As aforesaid, within this system, the doctor, in part because of the sheer convenience of it, possesses a flexibility of expression not shared by the patient they treat. The most immediate consequence of this privilege is the ability, both as a passive and active force, to determine which modes of communication are valid. Their epistemic privilege, then, is twofold; on the one hand, their knowledge is considered superior by default, both internally and externally, and on the other, they, alone, decide how the broader conversation develops

Consequently, Carel and Kidd use vocabulary reminiscent of social activism. This diction suggests that the issue is not merely

Havi Carel and Ian James Kidd, "Epistemic Injustice in Healthcare: A

Philosophical Analysis," Medicine, Health Care and Philosophy 17, no. 4 (2014): 531, 10.1007/s11019-014-9560-2. 
a philosophical (or scientific) one-consisting of abstractions and hypotheticals - but one of a specific, human importance: relevant, applicable, and necessary. In this way, "ill persons" are analyzed as a subset of disenfranchised people, and HCPs are deemed socioeconomically exalted, or "privileged."

Spread throughout Carel and Kidd's analysis of how a culture of medical professionals has continued to unjustifiably objectify their patients is the suggestion that what best characterizes a successful patient/doctor relationship is absolute trust given and received by both parties. When hermeneutical injustice occurs, and a patient is not given the tools by which to communicate their pain or their concerns, the lack of communication contributes to a lack of trust. Similarly, when a doctor disregards a patient's testimony (i.e., testimonial injustice) as frivolous or unprofessional, the patient is discouraged from providing testimony in general. Carel and Kidd write

But we might also find that even when the clinician's assumption of epistemic authority in relation to matter $\mathrm{x}$ is correct, the clinician's style of interaction is overly dismissive. Her disregard of the patient's perspective on $x$ might still be detrimental to the patient's well-being, not least since the judgment that one's testimonies have been disregarded tends to undermine one's ability and willingness to engage in further interpersonal exchanges. ${ }^{2}$

The clinician's style, irrespective of content, can damage the trust necessary for the doctor to adequately do their job, which is ensuring the mental and physical well-being of the patient.

Perhaps most intriguing is the authors' solution to the issue of epistemic injustice: the phenomenological toolkit. According to Carel and Kidd, "It provides a flexible individual tool which patients and clinicians can use to develop their understanding of their illness experiences. It includes three steps: [1] bracketing the natural attitude, [2] thematizing illness, and [3] reviewing the ill person's being in the world." 3 For Carel and Kidd, the patient's lack of trust in the self as well as their overwhelming "trust" for their doctor-although perhaps holy dread is a better term-coupled with the doctor's overwhelming trust in themselves and abundant lack of trust in their patient is the precise social climate that allows epistemic injustice to thrive. The best way, then, to undermine this process is for the patient to derive a specific meaningfulness from their ill state (a type of objectification: an ownership) and for the doctor to derive a specific meaningfulness from the patient (a type of subjectification: a letting-go). Taken together,

2 Carel and Kidd, "Epistemic Injustice in Healthcare," 531

3 Carel and Kidd, "Epistemic Injustice in Healthcare," 537-38. the three steps of Carel and Kidd's phenomenological toolkit allow the patient to experience their illness in a way that is comfortable and uninhibiting. It is assumed-because the authors do not discuss the toolkit from the doctor's perspective - that the medical professional, by virtue of having seen this phenomenon occur, is now made aware of the patient's actual ontology.

The toolkit's first step, bracketing the natural attitude, is designed to permit both the patient and clinician to observe the illness, not as a disease entity, but as a total experience. The focus, here as elsewhere, is on patient testimony - ultimately in the hopes that they may perceive the illness in less prescriptive ways. Thematizing the illness brings into question the various perspectives that HCPs, patients, and family members may have vis-à-vis the illness's identity function-e.g., what the HCP may see as malignant and objectively bad, the patient may see as an essential part of themselves. ${ }^{4}$ Carel and Kidd suggest that this step of the toolkit be applied via focus group. The final step, by way of practical application, encourages the patient to consider themselves as existing in the world in the newly-defined context of the disease experience. It is the culmination of the first two steps and occurs both individually and subjectively, i.e., independent of the HCP.

While the central thesis of "Epistemic Injustice in Healthcare" is for the most part exemplary, it does suffer from one major blindspot, inasmuch as it fails to address the actual source of the problem: it does nothing, or at least very little, to combat the epistemic privilege possessed by HCPs. In this vein, the paper would have benefited greatly from a clearer explanation of how the phenomenological toolkit directly adjusts the doctor's perception of the patient - the most lasting change, as it were, occurring from within.

\section{MY ARGUMENT}

Considering the risk/benefit of implementing any such toolkit for any such egalitarian purpose, the authors' assumptions regarding the existence of epistemic injustice against ill persons seem plausible enough to warrant reform of the kind described. The phenomenological toolkit, in permitting ill persons to express themselves, could potentially resolve both complaints, albeit in different ways. What is perhaps most concerning about the toolkit, however, is that it does not apply to the doctor's own subjective experience enough. While the problem of systemic epistemic injustice is sociopolitical, it is also philosophical, insofar as the epistemic imbalance results not only from a lack of trust, socioeconomic status, or even professional

4 Essential to this step is the juxtaposition of distinct, though not necessarily antithetical, viewpoints. 
authority, but also a lack of empathy. Both doctor and patient represent complex, subjective states of being, such that a truly useful forum for open communication ought to involve more than just an opportunity for the patient to speak and be heard. This means that the boundary of the phenomenological toolkit needs to be broadened.

As it stands, the first two of its three steps-bracketing the natural attitude toward illness and thematizing illness-do encourage some involvement from clinicians. Carel and Kidd suggest, in the former, that both doctor and patient, instead of believing in the disease entity itself, ought to learn to see the disease as it expresses itself through the patient's direct experience (e.g., through symptoms, fear of death, et cetera). Similarly, in explaining the second step, the authors recognize that, "[patients, family members, and health professionals] each will thematize an illness differently." ${ }^{5}$ It is the third step, however"reviewing the ill person's being in the world"- that isolates the ill person's experience from that of the doctor. ${ }^{6}$ What is more, each step can be improved by encouraging the patient to access the clinician's own subjective experience.

Because one's preconceptions are inextricably linked with one's sense of identity (or one's subjectivity), the only way for these preconceptions to be constructively challenged is for them to clash with another identity, forcing the objectification of one's own subjectivity, which naturally facilitates empathy (i.e., the acknowledgement of an equally valid subjectivity in someone else). This cannot be a passive experience. Carel and Kidd's undeveloped toolkit, even without the steps required to accomplish this, already has this goal in mind: "The toolkit is a patient resource, but it is also aimed at training clinicians. If clinicians are trained in this way and, consequently, become more open to patients' experiences and better able to interpret them, this would be yet another way to address the hermeneutical gap."

I suggest three amendments be made to better realize Carel and Kidd's goals: (1) bidirectional expression of intent, (2) formal mediation, and (3) posthumous review. (1) The doctor, in addition to the patient, will be invited to participate in each of the three steps of the phenomenological toolkit, not passively but actively. This means that both patient and doctor will be given the opportunity to reevaluate what it means to be ill, specifically in relation to the self. The doctor and patient will each reassess the disease entity, its thematization,

5 Carel and Kidd, "Epistemic Injustice in Healthcare," 538

6 Carel and Kidd, "Epistemic Injustice in Healthcare," 538

7 By "hermeneutical gap," Carel and Kidd are referring to the

communication gap caused by hermeneutical injustice; See Carel and Kidd, “Epistemic Injustice in Healthcare," 537. and also how these alterations affect each of their roles in the doctor/ patient relationship. In this renewed environment, the doctor will be encouraged, just like the patient, to express their beliefs/perspectives with respect to each of Carel and Kidd's steps. Unless the doctor subjectively experiences the patient subjectively experiencing the doctor, it runs the risk that the patient may feel understood without it actually being the case. This, as it were, treats the symptom but not the disease. (2) Formal mediation will include a trained professional supervising the dialogue between doctor and patient, ensuring that it is clear and symmetrical. The mediator will also document the implementation of the toolkit. It is important that the mediator approach the exchange free of bias. (3) The last step will include an uninvolved board of clinicians and volunteer patients/mediators reviewing all pertinent documentation, evaluating the overall efficacy of the exchange, suggesting improvements, and reinforcing effective techniques. This final step is significant in that it takes place after the toolkit has been implemented. Its aim is to improve the efficacy of future toolkits.

I am well aware that these suggestions involve a drastic reconfiguration of common healthcare practices, most of which will be rather expensive and time-consuming to implement, but — as many have said before me and are sure to say again — human equality, if anything, is worth the trouble. ${ }^{8}$

\section{COUNTERARGUMENT/OBJECTION TO MY VIEW}

Readers will notice two notable weaknesses in my thesis. The first addresses the efficacy of the model, and the second addresses its efficiency. In an attempt to legitimize the personhood of the patient to the doctor, I have suggested that the doctor's personhood concurrently be demonstrated to the patient. This requires that Carel and Kidd's phenomenological toolkit - the aim of which is to give the patient's word with respect to their illness more epistemic value, to the doctor and to themselves - extend itself out to the direct experience of the doctor. This runs the risk, however, of reestablishing, by default, the epistemic privilege experienced by the doctor (i.e., before the application of the toolkit). Taking into consideration that the ultimate goal of the toolkit is to lend a voice to the voiceless - in the form of phenomenological expression - if the doctor, who already possessed a rather deafening voice to begin with, is elevated in concert with the patient, then the old dynamic (i.e., of a doctor who systematically holds more epistemic authority than the patient) has simply been reinstated.

8 Training, recruiting, and paying full-time mediators is the most considerable of these administrative concerns. 
Injustice stems from an imbalance or an inequality. While it is certainly a point of contention to admit it, the only viable method of reinstating equality (i.e., correcting the imbalance) is by an act, however temporary, of inequality.

What is more, the application of my modified toolkit, which includes three steps-bidirectional expression of intent, formal mediation, and posthumous review - is almost impossible to implement unless at great financial cost to the healthcare industry. Because of its specific and formal structure - a quality that Carel and Kidd's unmodified toolkit lacks— the toolkit I propose requires immense administrative overhauls, redistribution of funds, and even the generation of a new career field. Unless it can be demonstrated that these changes are effective (e.g., in some sort of clinical trial) not to mention how they will be funded, the risks associated with making these changes outweigh the benefits.

\section{RESPONSE TO OBJECTION}

While these critiques are reasonable, they are founded upon incorrect assumptions and the misrepresentation of my overall argument. Left unchecked, Carel and Kidd's phenomenological toolkit fails to adequately address the doctor's perspective. My objective — different from theirs - is to correct not epistemic injustice, but epistemic privilege. To address the first critique, unless the doctor more actively participates in the patient's experience (regarding their own illness), like a conversation or a friendship, then this is impossible. The worst possible resultthough admittedly better than what we have right now-is the patient walking away having been forever changed, while the doctor remains the same. This is especially disconcerting when considering that the doctor must treat patient after patient. Claiming, as stated above, that the "only viable method of reinstating equality ... is by an act ... of inequality" presupposes two mistruths: that (1) systemic injustice rests on a twodimensional plane, and that (2) justice must be retaliatory.

While it can probably be said that privilege itself must be taken away from one group before it can be given to another, the redistribution of privilege is not our objective. We do not desire patient privilege; we desire doctor/patient epistemic equality. The doctor's perspective need not be lowered beneath that of the patient; the patient's perspective, instead, must rise to meet the doctor's. Despite my confidence in this motion, the second step of my modified toolkit is specifically designed to address this issue, to ensure that epistemic justice prevails in each and every doctor/patient interaction. By the introduction of an impartial third-party (ideally not medically trained but rather professionally trained in mediation), the "old dynamic" mentioned above will be avoided.

In forming my thesis, I anticipated the possibility that these ideas may be overly ambitious and difficult to implement without adequate preparation. While this remains true, this does not, however, prove that they will not be effective or that they should not be attempted. The medical industry is incredibly lucrative, and while its resources are limited, my modified toolkit contains, if desired, a new field of expertise: doctor/patient mediation. This could have far-reaching implications not only on modern medicine, but also on many aspects of society - education, economics, and psychology - the least of which may be wealth creation. As a final note, the gradual application of these principles, if necessary, could better facilitate their total implementation.

\section{CONCLUSION}

As we have seen, epistemic injustice derives from a systemic prejudice against ill persons within the medical profession. Its source, epistemic privilege, is a result of (1) HCPs overestimating the value of their own knowledge, (2) HCPs underestimating the value of their patients' knowledge, (3) patients overestimating the value of their doctors' knowledge, and (4) patients underestimating the value of their own knowledge. Because an undue lack of trust has caused and continues to perpetuate this issue, restoring this trust ought to be our main objective.

In this paper, I have analyzed and supplemented Carel and Kidd's alleged solution to epistemic injustice, the phenomenological toolkit, with some additional steps of my own. The purpose of these steps is to extract the doctor's own perspective on existential issues, so as to elicit a clash of consciousness. This clash-perhaps best summarized as a moment of epiphany during which a subjective being becomes aware of their own objectivity in addition to the subjectivity of someone else-is meant to facilitate empathy. Once this empathy has been allowed to thrive, an environment rife with open communication will restore the trust that epistemic injustice, by way of epistemic privilege, had destroyed.

Because these notions are theoretical only, and because they could, if implemented, restructure an industry that usually resists change, I recommend that researchers in the field undergo extensive experiments to validate my hypotheses. These experiments should include oneon-one discussions between doctors and patients, both addressing their individual roles in the process, with the assistance of a thirdparty mediator, likely being filled by a volunteer during the beginning 
stages of the experiments. Researchers should then conduct individual interviews with each of the three participants, observing, if possible, a correlation between the clinician's openness and the patient's level of trust. These qualities should be tracked with an assigned number value-e.g., 1 for "not significant" and 5 for "very significant."

As I have stated previously, the doctors of today — perhaps, tragically, without knowing it — have a vested interest in the successful eradication of epistemic privilege. The true healers-those who have taken the

Hippocratic Oath in earnest, those who serve the public out of compassion and not economic or social prestige- must admit that a lack of trust between themselves and their patients, especially from their patients, inhibits the doctor in their quest to heal the sick. It must be admitted, then, that this system, in its current form - that which treats its patients like objects, that which concedes to the disease entity more humanity than the person afflicted - is inimical to the core philosophy of modern medicine. As we have seen throughout history, social change is not easily obtained. What is more, it is often difficult to imagine how necessary social change is until we have already grown accustomed to the ways in which it has improved society. We are, then, indebted-now not unlike then - to those among us who are able to see the wind before the storm. Might there, then, be no better assessment of a society's compassion than the way it treats the weakest of its citizens?

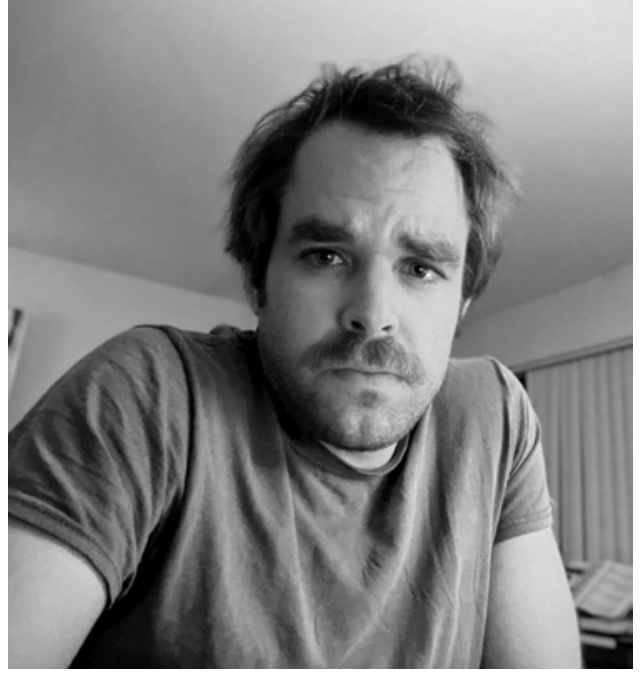

Bradley Holder is a senior English and philosophy major at the University of California Irvine with a minor in creative writing. Because he believes that art is philosophical and that philosophy is artistic, his passion for one only informs the other. Lately, he's been reading a lot of Søren Kierkegaard. 